\title{
AN INTEGRATED APPROACH TO RELATE HOT FORGING PROCESS CONTROLLED MICROSTRUCTURE OF IN718 AEROSPACE COMPONENTS TO FATIGUE LIFE
}

\author{
Michael Stoschka ${ }^{1}$; Martin Riedler ${ }^{2}$; Martin Stockinger ${ }^{2}$; \\ Hermann Maderbacher ${ }^{1}$; Wilfried Eichlseder ${ }^{1}$ \\ ${ }^{1}$ University of Leoben; Chair of Mechanical Eng.; Franz-Josef-Str. 18; 8700 Leoben; Austria \\ ${ }^{2}$ Böhler Schmiedetechnik GmbH\&Co KG; Mariazellerstraße 25; 8605 Kapfenberg; Austria
}

Keywords: Fatigue analysis, Thermo-mechanical processing, Aerospace components, Microstructure characterization.

\begin{abstract}
The evolution of microstructure of hot-forged superalloy 718 can be tailored to specific customer demands by local adjustment of the overall metallic forming process. Further on, increased economic sustainability will continuously comply with the light-weight demands. Therefore it is necessary to incorporate the local fatigue behavior right during the design stage.

The main output of hot-forging process simulation is defined by microstructural parameters like grain size, amount of $\delta$-phase as well as $\gamma^{\prime(")}$ - precipitation contents, etc.

The presented work shows the use of an alternative microstructural approach leading to two ancillary microstructural parameters called 'microstructural energy parameter $e$ ' and 'factor of heterogeneity $b$ '. This newly developed microstructural evaluation model, which is based on interpretation of the cumulated shape of individual grains, supports an alternative characterization method of microstructure, encompassing morphological information in a combined manner. Based on the thoroughly used microstructural based energy approach it is possible to close the complex simulation chain between forging process simulation and fatigue.

The developed method to assess a closed simulation loop at design stage is based on extensive fatigue tests and corresponding metallographic work. This leads to a parametric interface between the individual project tasks. The basic approach presented here establishes a common link between hot forging simulation codes and calculation of the component life time for superalloy 718 .
\end{abstract}

\section{Introduction}

To describe a forging process with the aim of linking fatigue life approach to microstructure, numerous relevant process influenced factors must be considered. The presented methodology shows the generation and use of a microstructural based evaluation method to link the grainshape based texture and morphology to fatigue.

The obtained microstructure is described further on, by two new parameters; namely the microstructural energy parameter $e$ and the factor of heterogeneity $b$. The microstructural energy parameter is linked to the average grain size and the factor of heterogeneity is a numerical representation of the degree of bimodality focusing on coarse grain regions.

Extensive experimental data from former specimens fatigue tests contribute extensively to correlate hot-forging process dependent microstructure to fatigue life. In addition the fatigue behaviour of specimens manufactured also out of different forgings is considered. The fatigue 
life behavior of hot-forged superalloy 718 is assessed by local SN-curves using parameters like fatigue limit $\sigma_{T}$; slope $k$ and number of cycles to fatigue limit $N_{T}$.

To achieve the life time distribution tendency of the hot-forged part already at early design stages by the use of simulation tools, the microstructural evolution is predicted numerically using the model introduced by Stockinger et.al.. As extension to the introduced model both the mean grain size and the grade of bimodality will be derived from the microstructural parameters numerically using process dependent evolution of local forging parameters such as true strain, grade of recrystallisation, change of mean grain size, etc.

\section{Methodic Approach}

To predict the local lifetime of a forged part, it is necessary to define meaningful interface parameters between numerous disciplines. Figure 1 shows the global flowchart to establish a closed simulation chain for hot-forged superalloy 718 parts.

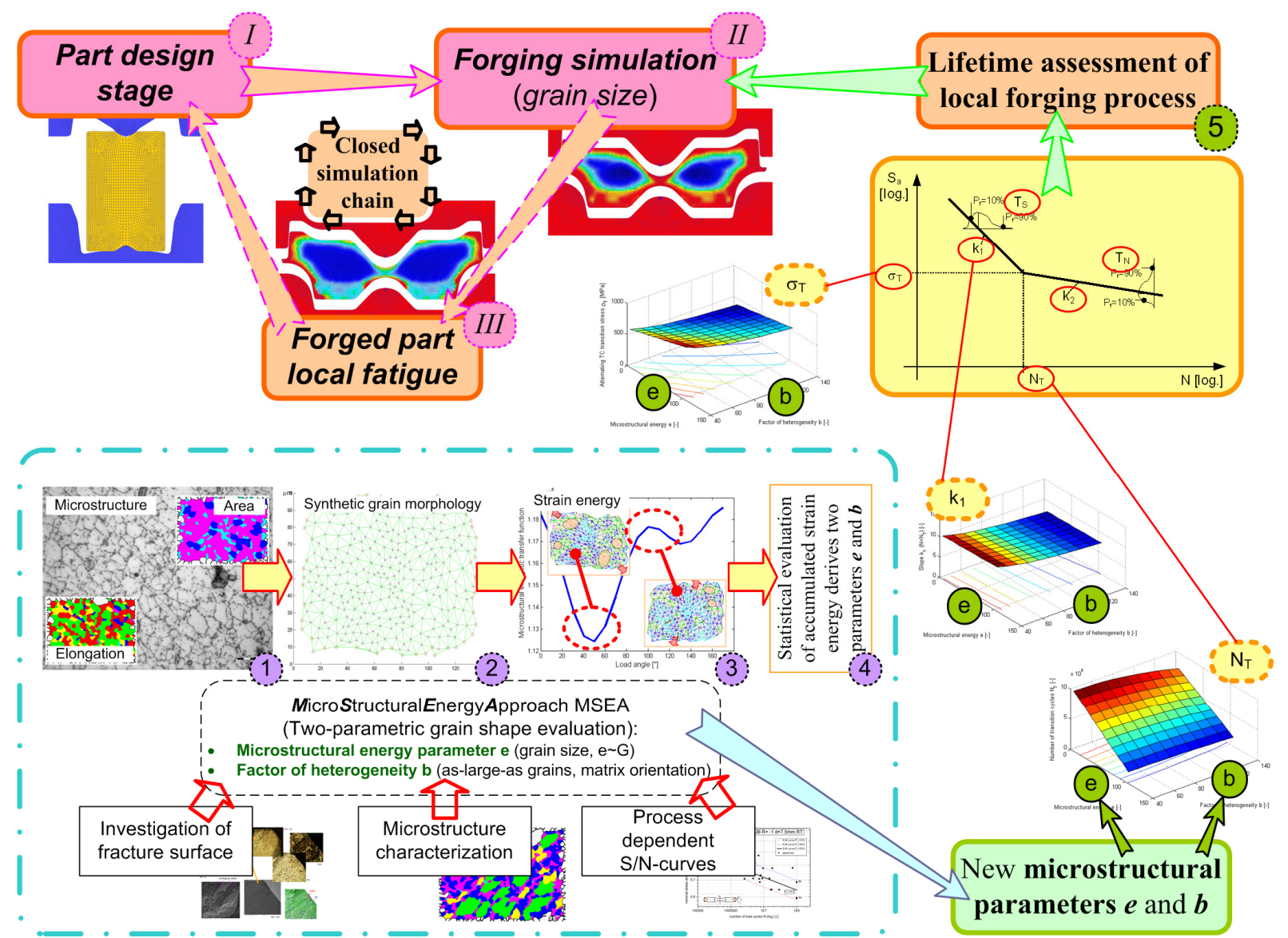

Figure 1. Flowchart of a holistic microstructural based fatigue approach for superalloy 718 .

First; the forging process has to be set-up to fulfill the requirements of the customer like part shape and average grain size at design stage. This can be done by using advanced simulation tools like Deform ${ }^{\circledR}$ enhanced by extensive user-defined subroutines to predict the microstructural evolution. Second; fatigue data covering a wide range of hot-forging processparameters deliver a multi-parametric fatigue matrix. As final third step; the two parameters $e$ and $b$ are introduced to link both the complex results of hot-forging part simulation and microstructural dependent local lifetime behavior. To explain the microstructural energy parameter $e$ and the factor of heterogeneity $b$ a short summary of the MicroStructural Energy Approach ("MSEA") is given. 


\section{MSEA Survey}

The demand of simultaneously characterizing the grain-shape based microstructure of plane metallographic sections possessing uniformly distributed grains and bimodal microstructure can not be reached by considering just the average ASTM grain size. The microstructural formation which includes grain boundary morphology and $\delta$-phase behavior influences forged superalloy 718 fatigue life in major ways [1-6]. Therefore, a two-parametric alternative method was developed in a previous work to gain additional grain shape information [7].

A detailed particle-shape based examination was performed for each metallographic section. The microstructure assessment embraces not only information about the shape of the grains, but also the amount of twins and $\delta$-phase. From the images taken, grain boundaries were transformed into synthetic binary grains restraining carbides and twins. Figure 2 shows three different metallographic sections with mean ASTM grain sizes ranging from $G=12.5$ down to $G=7.5$ but extreme differences in amount of as-large-as grain und microstructural texture. The evaluation of the mean grain size was done using Abram's procedure for non-equiaxed microstructures. A smaller grain size is represented by increase of the microstructural energy parameter $e$; whereas the presence of contorted large grains is indicated by a high factor of heterogeneity $b$. The formation of such large grains can be driven by secondary grain growth for instance [8].

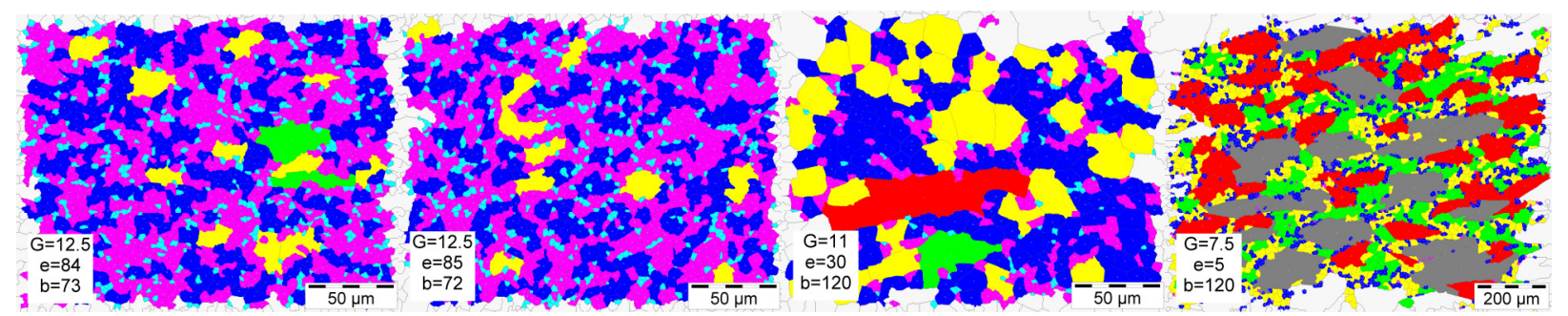

Figure 2. Evaluation examples of hot-forged superalloy 718 metallographic sections.

The analyzed properties included planimetric measures like area, equivalent circle diameter as well as advanced geometric properties like form factor, elongation, convexity, outerior diameter, interior extend and cord length. To achieve independent grain-shape based geometric properties, a true factor analysis was done. It transforms the possibly correlated grain properties like diameter, convexity, shape factor and others into a set of uncorrelated principal components which are shown in the left subfigure of figure 3 as spatial vectors.
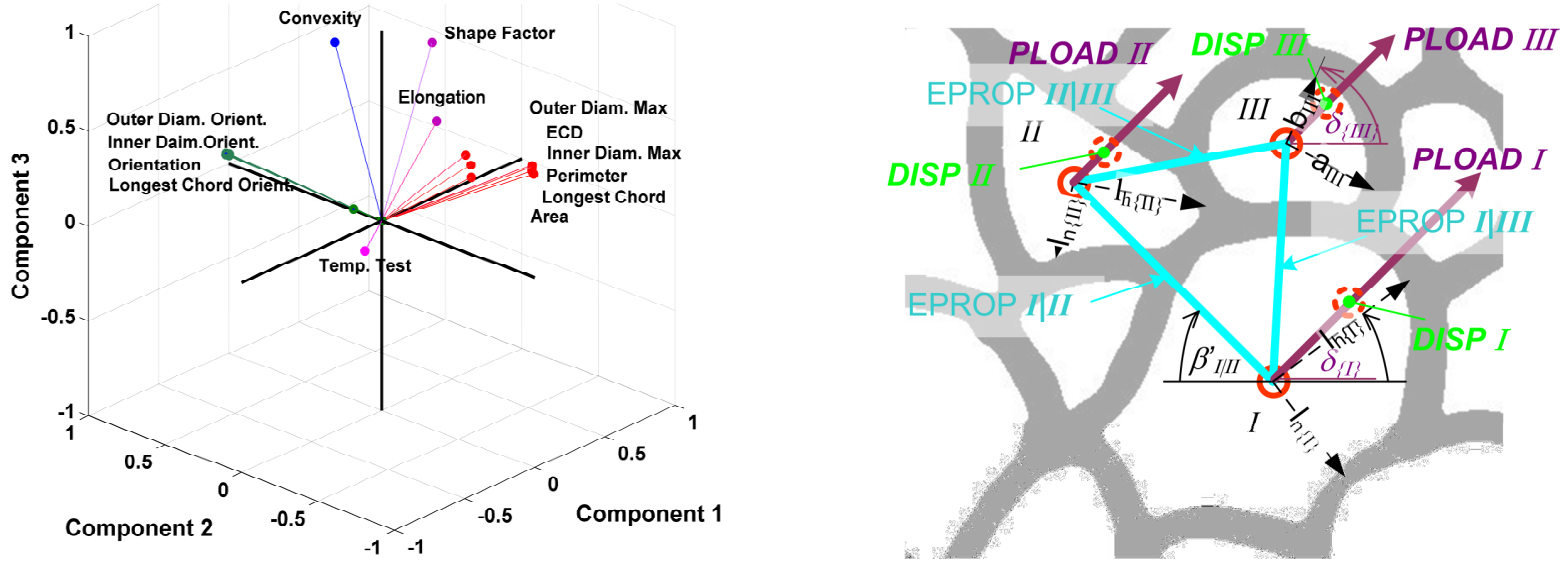

Figure 3: Spatial vector results based on grain-shape factor analysis [9] (left);

Homologous model of interacting grains [7] (right). 
Thus, an assignment by evaluation of principal component direction can be done. In addition, the impact magnitude is reflected by the principal component norm. Major independent properties are therefore the length of the longest chord, the orientation vector and finally the shape of the grain reflected by convexity and elongation.

Additionally, the relationships of the particular grains are included, leading to an adjacency matrix implemented as synthetic grain morphology. The connectivity and the particle-based grain parameters allow the implementation of homologous unit-cell models. The geometric properties of individual, but adjacent linked grains, are represented in the material property card of the accordant beam element; see right subfigure of figure 3. Due to combining results of grain morphology and individual grain-shape parameters in this way, loading the synthetic plane model by unit displacement and evaluating the strain energy distribution, a stiffness transferfunction of unit-cells is achieved. Examining the transfer function statistically by the use of finite element beam models, the microstructure parameters $e$ and $b$ are derived. The microstructural energy is calculated by the average of the transfer function energy $e$, whereas the factor of heterogeneity $b$ describes the function curve divergence. The whole determination process is implemented as semi-automatic microstructural evaluation user-defined analysis ${ }^{\circledR}$ toolbox. Because of the microstructural energy $e$ is strongly controlled by the equivalent circle diameter, the following equation defines the relation to the ASTM grain number $G$.

$$
e(G)=A+B e^{C G}
$$

Table I. Parameters to link microstructural energy $e$ and grain number $G$ exponential.

\begin{tabular}{|c|c|c|}
\hline $\mathrm{A}[-]$ & $\mathrm{B}[-]$ & $\mathrm{C}[-]$ \\
\hline 0.1 & 0.014 & 0.706 \\
\hline
\end{tabular}

The microstructural energy parameter $e$ varies in the range of about $2<e<145$; whereas the corresponding grain size number is about $7<G<14$. It has to be stated that the parameter $e$ is sensitive to fine-grained microstructures and is hence most suitable for hot-forging applications.

\section{Experimental Work}

Fatigue results, both of specimens taken out of pan-cakes and of aircraft part cut-ups, have been used to define the interrelationship between hot-forging process parameters and life time behavior. The investigated fatigue specimen test series out of pan-cakes ranges from alternating stress condition with different stress gradients up to tumescent testing with uniform stress distribution. Due to the extensive hot-forging process parameter study the microstructure varies in a broad range leading to significant deviation regarding fatigue behavior.

For the establishment of a throughout fatigue assessment, it is necessary to choose a reference value which is accessible both in regular shaped specimen's and complex forging parts. A stress based local solution is achievable by using the notch stress and the corresponding stress gradient. The evaluation of the executed test series is therefore done using the notch stress amplitude as well as the corresponding stress ratio and the stress gradient. The normalized results in figure 4 are related to fatigue notch stress amplitude of raw-material at about one million cycles. Both pictured S/N-curves show extreme different fatigue life behavior, varying slopes and scatter bands. The S/N-curve shown in the right subfigure of figure 4 defines for instance the optimization goal as maximum obtainable local fatigue stress for the complex forged aircraft part, especially at the alternating high-loaded regions. 

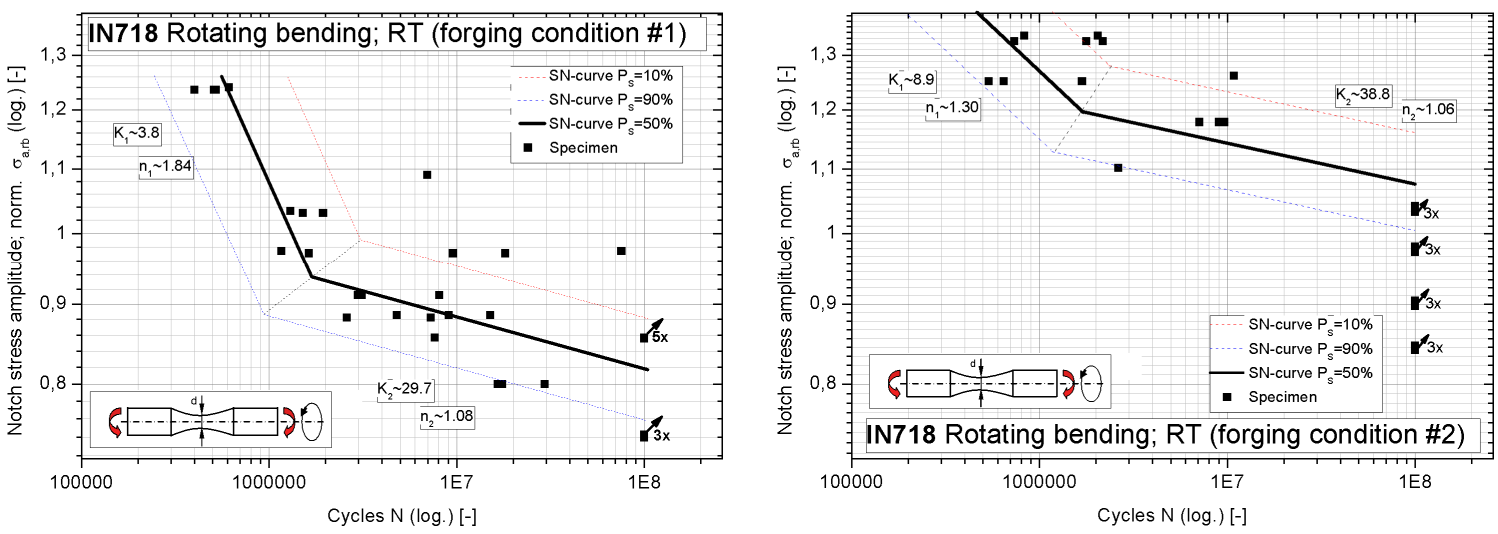

Figure 4. Excerpt of SN-curve test results of superalloy 718 pan-cake specimens.

The test program implies smooth and notched specimens to characterize notch effects as fatigue factor $n$. The fatigue factor $n_{\chi}$ as dependency of the stress gradient $\chi^{*}$ is defined according to [10] as exponential law using characteristic stress gradient values; for example in the case of fatigue experiments ranging from tension-compression to bending tests.

$$
n_{\chi}\left(\chi^{*}\right)=1+\left(\frac{\sigma_{b d, a}}{\sigma_{t c, a}}-1\right)\left(\frac{d \chi^{*}}{2}\right)^{K_{D}}
$$

The ratio between alternating bending stress $\sigma_{b d, a}$ and alternating tension-compression stress $\sigma_{t c, a}$ is material dependent and characterizes the supporting effect capability. The equivalent specimen cross section diameter is defined as $d$, the exponent $K_{D}$ is material dependent and defines the slope of the exponential function. Due to the change of the transition stress point as shown in figure 4 , a basic evaluation of the fatigue factor was done in the fatigue region first. Table II shows the corresponding values for an evaluation point of six hundred-thousands load cycles.

Table II. Fatigue parameters of superalloy 718 using a stress gradient approach.

\begin{tabular}{|c|c|c|}
\hline$\sigma_{\mathrm{bd}, \mathrm{a}} / \sigma_{\mathrm{tc}, \mathrm{a}}[-]$ & $\mathrm{b}[\mathrm{mm}]$ & $\mathrm{K}_{\mathrm{D}}[-]$ \\
\hline 1.15 & 7.5 & 0.17 \\
\hline
\end{tabular}

As an additional challenge the influence of different fatigue test temperatures has to be considered in the model ranging from 20 up to $650^{\circ} \mathrm{C}$. It has to be stated that the bigger part of data points are available at temperatures up to $150^{\circ} \mathrm{C}$.

From all SN-curves specimens metallographic sections have been taken with great care to achieve microstructures representing the samples. The metallographic assessment was done using the MSEA-approach. Figure 5 shows the complex arrangement of the microstructural investigated specimens regarding fatigue and grain distribution without microstructural based arrangement. 

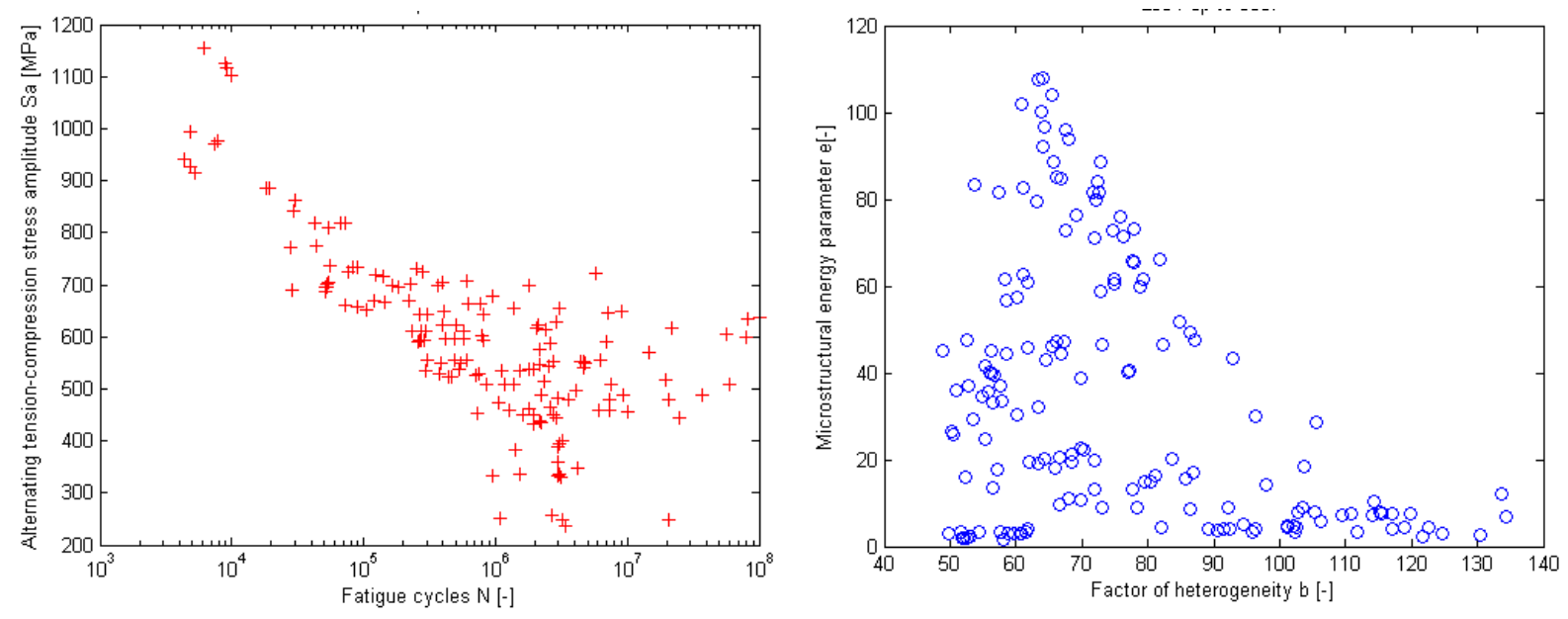

Figure 5. SN-fatigue scatter versus microstructural gridding (shown by change of $e$ and $b$ values concerning specimen evaluation up to $150^{\circ} \mathrm{C}$ ).

To link microstructural and fatigue results it is necessary to use a parametric definition of the $\mathrm{SN}$-curve. Therefore, a two-slope model was chosen for fatigue assessment. The alternating transition stress $\sigma_{T}$, the number of transition cycles $N_{T}$, slopes $k_{1}$ and $k_{2}$ as well as the scatter band values have been determined as functions both of microstructural parameters $e$ and $b$ by numerical variation. Figure 6 summarizes the dependency of the fatigue parameters $\sigma_{T}, N_{T}$ and $k_{1}$ as function of the microstructural style.
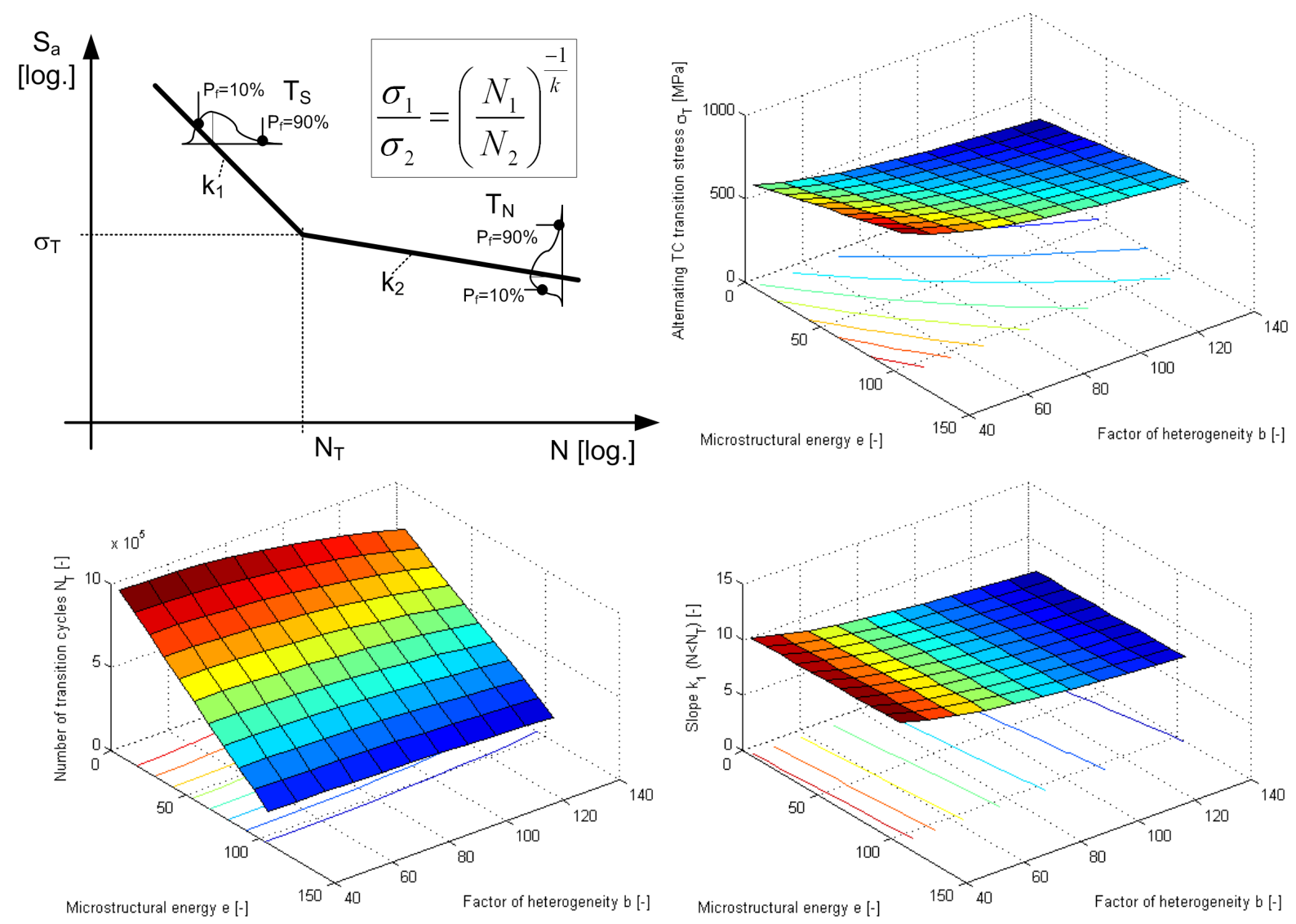

Figure 6. Link between fatigue and microstructural evolution of superalloy 718 up to $150{ }^{\circ} \mathrm{C}$ 
The presented relationship between $\mathrm{SN}$-curve and microstructural parameters was established by empirical and variational calculus and finally implemented as user-defined Matlab®-function. In compiled mode, as an executable file, it can be easily implemented in finite element codes like Deform ${ }^{\circledR}$ using their Fortran-interface.

Grouping the specimen data into microstructural clusters covering only a span between extreme values, it is possible to visualize the shift of the SN-curve as follows. Three subfigures in figure 7 represent the microstructure cluster regions and their trend in fatigue. The plotted data is based on specimen fatigue results and microstructural characterization using the introduced MSEA-technique.
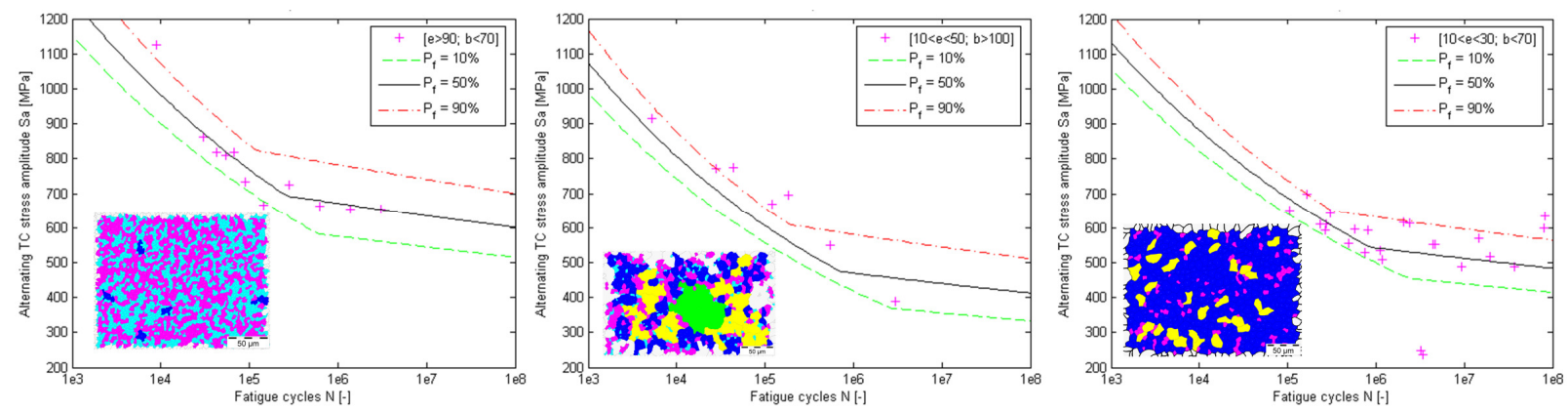

Figure 7: Fatigue tendencies on coarsening microstructure $(G \sim 13,11,10)$ up to $150{ }^{\circ} \mathrm{C}$

The group classification indicates that an increased bimodality improves the fatigue behavior somewhat; as in the case of a fine grain seam surrounding an insular large one. Nevertheless; extensive independent change of microstructural energy $e$ influences the specimen lifetime significantly. This impact of local different microstructure on fatigue is shown in figure 8 whereat the homogeneity of the fine grain structure and large grain amount is counteracting; reflected by contrary change of parameters of $e$ and $b$. In this special case, the difference in life time can be extensive although the mean ASTM grain number $G$ is at an equivalent level according to the evaluation of standard interception counting. To visualize the grain properties, the equivalent circle diameter and the particle based form factor are sketched also within figure 8 . The equivalent circle diameter correlates to the grain size. The form factor describes the deviation of the individual grain shape to the ideal geometric circle.

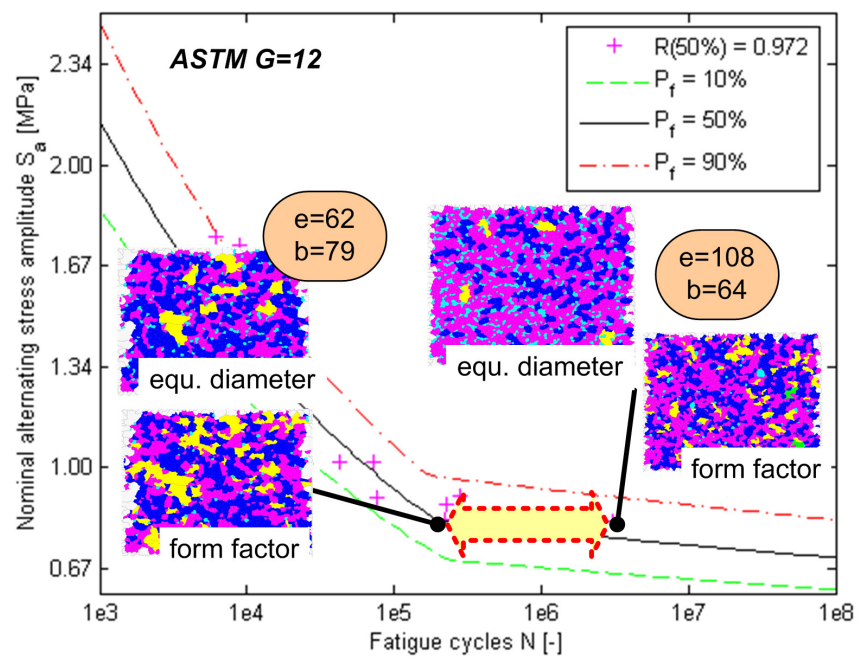

Figure 8. Load cycle number influenced by change of microstructure from coarse to fine grain and increased homogeneity. 
By using the two parameters $e$ and $b$, it is possible to quantitatively assess the microstructural dependent fatigue behavior of forged superalloy 718. The 'mapped' SN-curve results exhibit a dramatically reduced scatter band in comparison to the 'raw' input data; see Figure 5.

\section{Model Enhancement}

The numerically derived correlation between microstructural parameters $e$ and $b$ and fatigue life is validly extended to temperatures up to $650^{\circ} \mathrm{C}$. Due to the $\gamma^{\prime}$-abnormality, the yield strength increases up to a temperature of about $800^{\circ} \mathrm{C}$. The fatigue strength in the long-life region is higher than at elevated temperatures, which is more remarkable at low stress levels close to the fatigue limit [11]. Based on the fatigue parameters $\sigma_{T}, N_{T}, k_{l}$ - which describe the change of the local SN-curve - a non-linear temperature influence is obtained. Life time results of specimens taken out of forged aircraft cut-ups have been mainly used to extend the database. The microstructural assessment was carried out again using the MSEA-approach. Therefore, only the temperature influence has been added to the user-defined Matlab ${ }^{\circledR}$ function. The heading of the implemented function is shown later on. The current interface uses the following input parameters do determine the endurable fatigue stress at a forced number of load cycles. Output of the function is the expected fatigue stress level $S_{a}$.

$$
\begin{aligned}
& S_{a}=\text { evalk1k2sTNT_L } 2334(N, e, b, P f, T, R, \text { chi }) \\
& N \text {... Number of load cycles [-] } \\
& e \text {... Microstructural energy parameter [-] } \\
& b \text {...Factor of heterogeneity [-] } \\
& \text { Pf ...Failure probability [\%] } \\
& T \text {...Temperature }\left[{ }^{\circ} \mathrm{C}\right] \\
& R \text {... Stress ratio [-] } \\
& \text { chi ... Stress gradient }\left[\mathrm{mm}^{-1}\right]
\end{aligned}
$$

If it is necessary to determine the endurable number of load cycles at a given local load situation; defined by the stress amplitude $\sigma_{a}$; one can minimize the load cycle error by using a function handle to the MSEA-fatigue function shown in equation (3). The displayed pseudo code complies with the Matlab syntax. The output here is the corresponding number of load cycles $N_{a}$ at the specified local node-based load conditions as defined by stress amplitude $\sigma_{a}$, stress ratio $R$ and stress gradient $\chi$.

$$
N_{a}=\text { min_function }\left(@(x) \operatorname{abs}\left(\text { evalk } 1 k 2 s T N T \_L 334(x, e, b, P f, T, R, c h i)-c_{a}\right)\right)
$$

The application of the MSEA-fatigue function provides a parametric link between microstructure and life time; see mark five in figure 1. To display the developed relationship in an abbreviated and compact form, the MSEA-function predicted stress amplitude is sketched over the fatigue test results. The match of predicted fatigue stress is shown graphically; arranged by temperature classes. All investigated specimens - both from pan-cakes and cut-ups - have been taken into account. The used forged components cover both structural aircraft parts and turbine disks. The experimental fatigue stress is drawn on the axis. The predicted stress values using the MSEAfatigue function are plotted on the ordinates of figure 9 to figure 11. 
Although at times, huge differences exist between predicted and tested lifetime; the general trend between MSEA-based prediction and tested fatigue values are acceptable for the basic approach presented here. The class dependent deviations can be explained on one hand by the 'limited' number of fatigue test cycles as well as the partial presence of low-cycle-fatigue specimens with high plastic content. On the other hand, the position of the evaluated metallographic sections does not match entirely with the specimen arrangement in the case of huge and complex forged cut-ups. Finally, the experimental work has been done at different test laboratory sites which also influence these results.
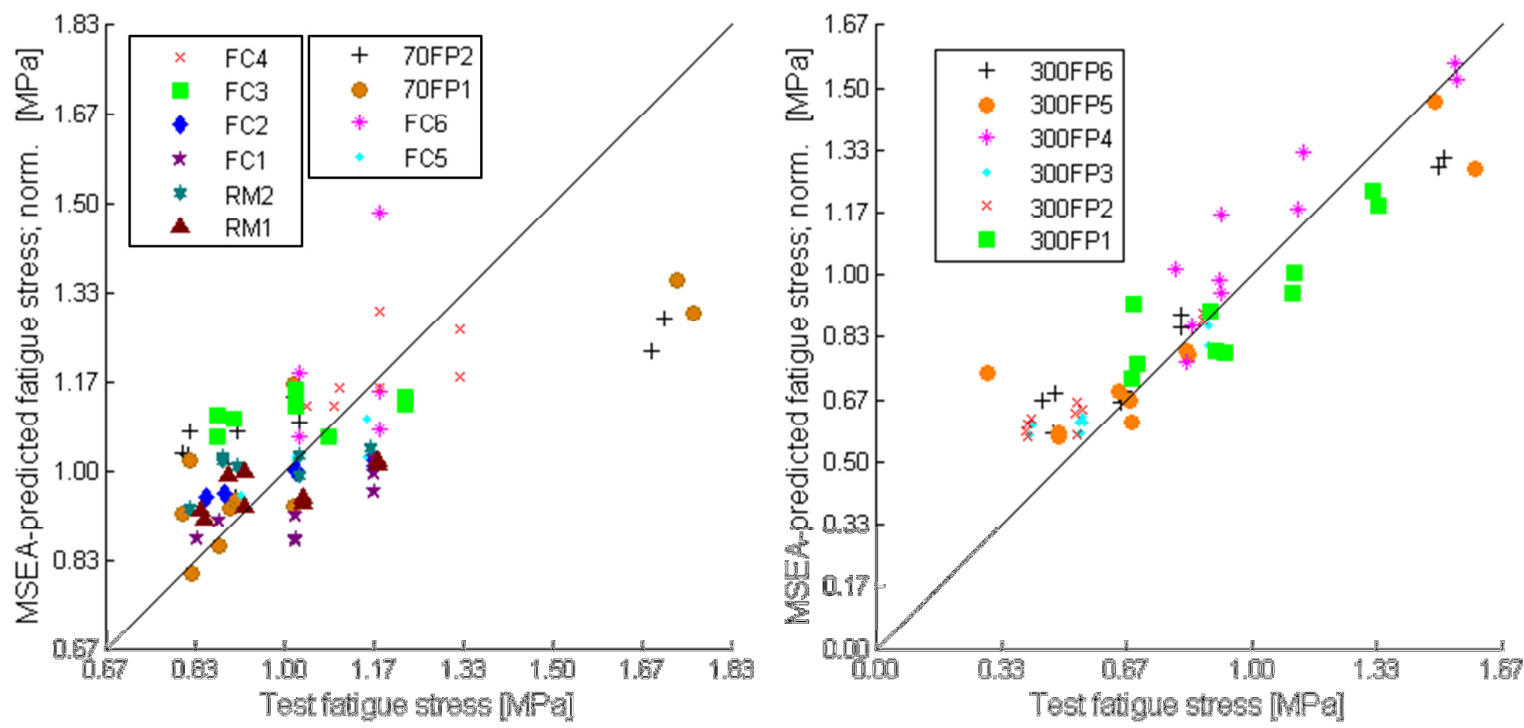

Figure 9. Match of predicted and tested fatigue behavior (test condition at $20^{\circ} \mathrm{C}$ and $150{ }^{\circ} \mathrm{C}$ )

The predicted and tested life time matches with sufficient accuracy under test conditions below $150{ }^{\circ} \mathrm{C}$. The mean trend is confirmed in both simulation and test. The specimen taken out of raw material are labeled RM, FC denotes specimen with specific forging condition in the left subfigure of figure 9 .
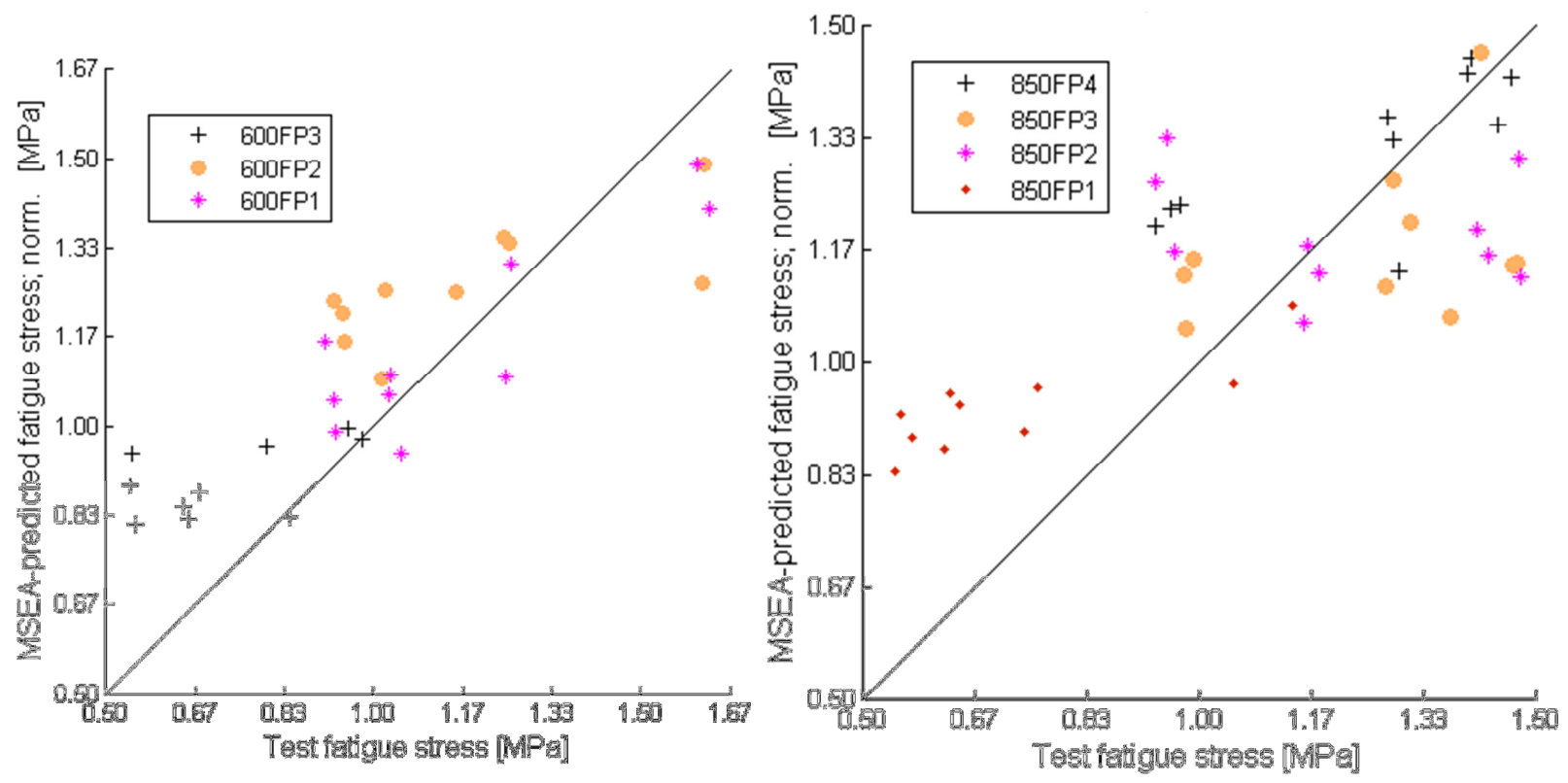

Figure 10. Match of predicted and tested fatigue behavior (test condition at $315^{\circ} \mathrm{C}$ and $450{ }^{\circ} \mathrm{C}$ ) 
The specimens taken out of forged aircraft components and turbine disks are for instance labeled as 70FP1, which corresponds in this case to test temperature of seventy Fahrenheit and part number one respectively. The subsequent legends in figure 9 to figure 11 are concatenated as character strings containing the test temperature and an ongoing index.

At temperatures between $300{ }^{\circ} \mathrm{C}$ and $500{ }^{\circ} \mathrm{C}$, a quite huge scatter band is observed. This can be caused by the quite high local plastic strain gradient at several specimen positions from different cut-ups. This leads to major variation in grain distribution at the 'single' metallographic sections investigated. Both, the lack of statistical assessment in case of the existence of a single piece of metallographic section and, the tendency of evolution of plastic strain due to differences in the location from where the specimen was taken from could be responsible for the measured deviations.
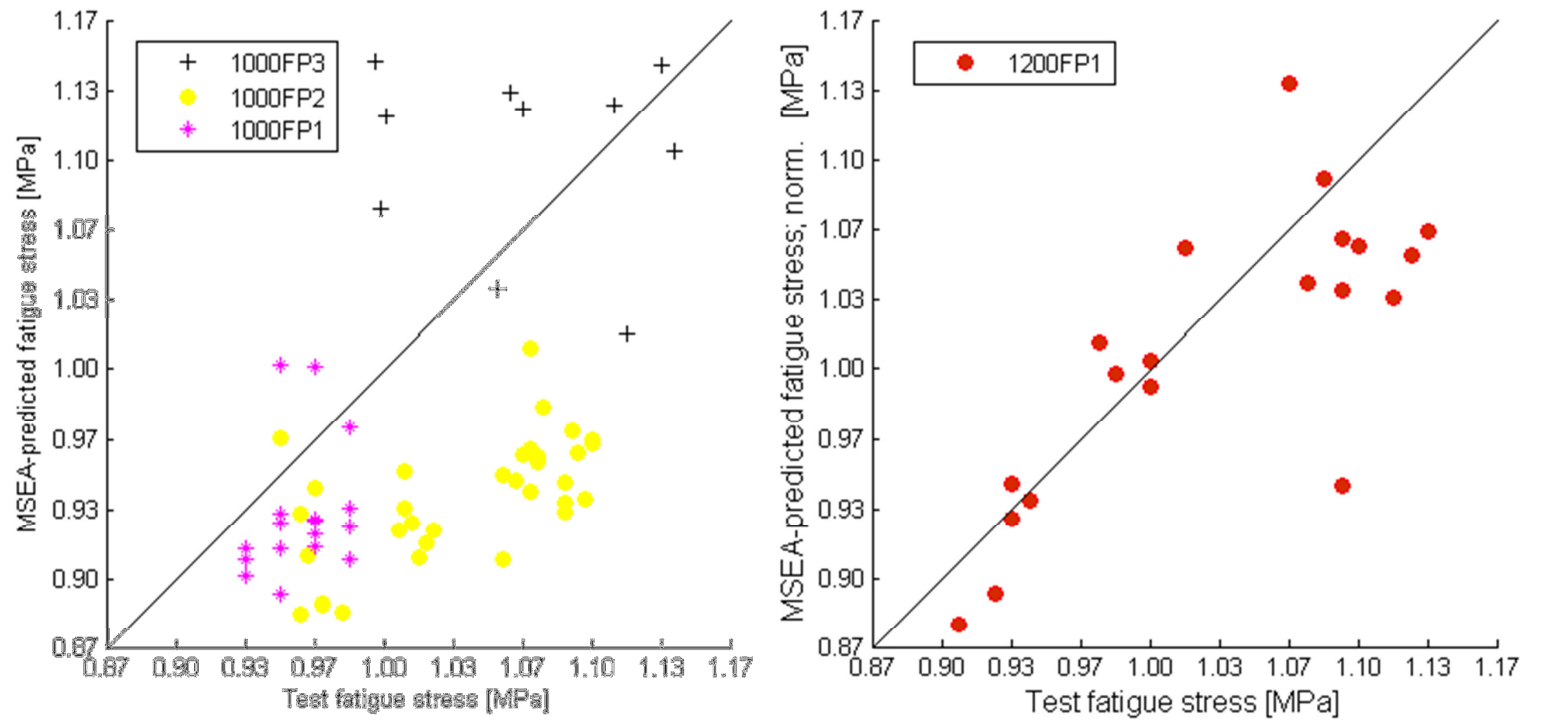

Figure 11. Match of predicted and tested fatigue behavior (test condition at $540{ }^{\circ} \mathrm{C}$ and $650{ }^{\circ} \mathrm{C}$ )

Finally, tests at temperatures above $500{ }^{\circ} \mathrm{C}$ contain a quite high plastic strain at cyclic tests which make it especially difficult to assess a proper stress range. Therefore, the stress amplitude of these tests was assessed at $\mathrm{N}_{\mathrm{f}} / 2$. This procedure is in accordance when evaluating low-cycle fatigue tests. Additionally, a mismatching of the predicted values compared to tested life time may be caused by the occurrence of run-through specimens at stress regions close to the endurance limit. Nevertheless, the basic MSEA-approach introduced here can be used to predict stress controlled life time based on the metallographic characterization using both the microstructural energy parameter $e$ and the factor of heterogeneity $b$ in the entire range.

\section{Closed Simulation Chain}

An overview over the currently used simulation process is given in figure 12 . The flowchart is structured into different program modules which perform user-specific tasks. The metal forming simulation tool Deform ${ }^{\circledR}$ currently defines the basic software. Substantial extensions with regard of grain growth evolution were added as Fortran subroutines to implement the microstructural model introduced by Stockinger $[12,13]$. This defines the simulation background for the following extensions. 


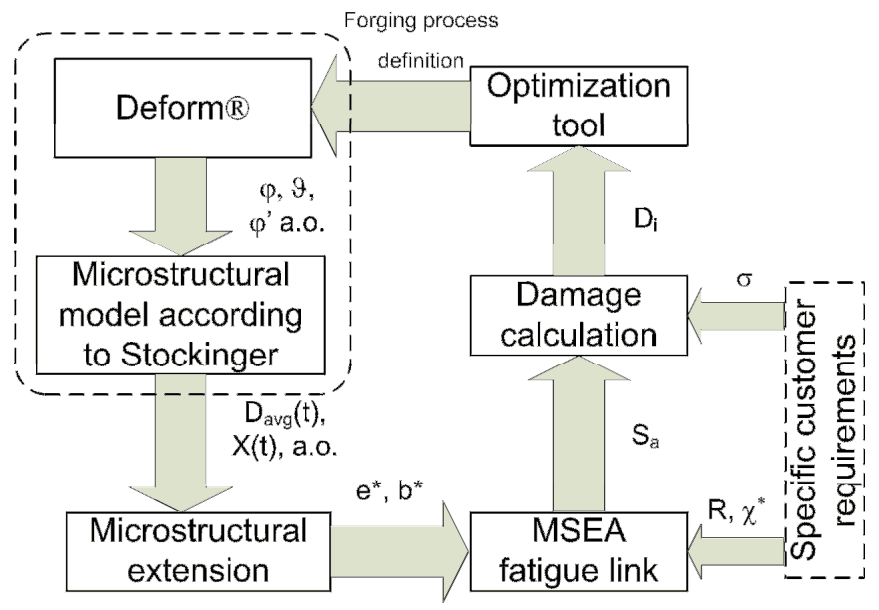

Figure 12. Flowchart of closed simulation chain featuring MSEA-interface values.

Firstly, the simulated microstructure has to be characterized using the MSEA-values $e$ and $b$ without the existence of a plane metallographic section to evaluate. The simple exponential equation (1) introduced earlier defines a relation between microstructural energy parameter $e$ and mean grain size number $G_{\text {avg }}$. After some iteration work it was found that it is necessary to alter the basic relation by a function involving the evolution of recrystallisation grade and plastic strain. Thus, the corrected microstructural energy parameter $e^{*}$ is achieved purely by simulation.

$$
\begin{gathered}
e^{*}\left(G_{a v g}, X(t), \varphi(t)\right)=\frac{e\left(G_{a v g}\right)}{f_{1}(X(t), \varphi(t))} \\
f_{1}=\left(\sqrt{\frac{1}{T} \int_{j}(1-X j)^{\varphi_{j}} d t}+C_{1}\right)^{C_{2}}
\end{gathered}
$$

Furthermore, the corrected factor of heterogeneity $b^{*}$ must be also derived from the microstructural simulation process parameters. Obviously, local recrystallisation behavior influences the occurrence of coarse grain structures and hence, has to be taken into consideration. In addition, the simulation step based growth in local grain size also influences the bimodality.

$$
\begin{gathered}
b^{*}(\Delta D(t), X(t), \varphi(t))=C_{3} \frac{f_{1}(X(t), \varphi(t))}{f_{2}(\Delta D(t))} \\
f_{2}(\Delta D(t))=\sqrt{\frac{1}{T} \int_{j} \Delta D_{j}(t) d t}
\end{gathered}
$$

These initial empirical findings are employed for an abstract axisymmetrical hot-forged spool made of superalloy 718. The Deform ${ }^{\circledR}$ calculation is carried out as two-dimensional simulation run. The expansion of microstructural properties through equation (5) and equation (6) leads to $e^{*}$ and $b^{*}$. At the development stage of the closed simulation chain, the user-operated data transfer to gain the corrected microstructural parameters was achieved by tracking individual points manually. The results presented show a coarse mapping of about 135 points, which will be automated by calling appropriate subroutines in the near future. Thereby, MSEA-fatigue results with finer resolution could be achieved without additional effort. 

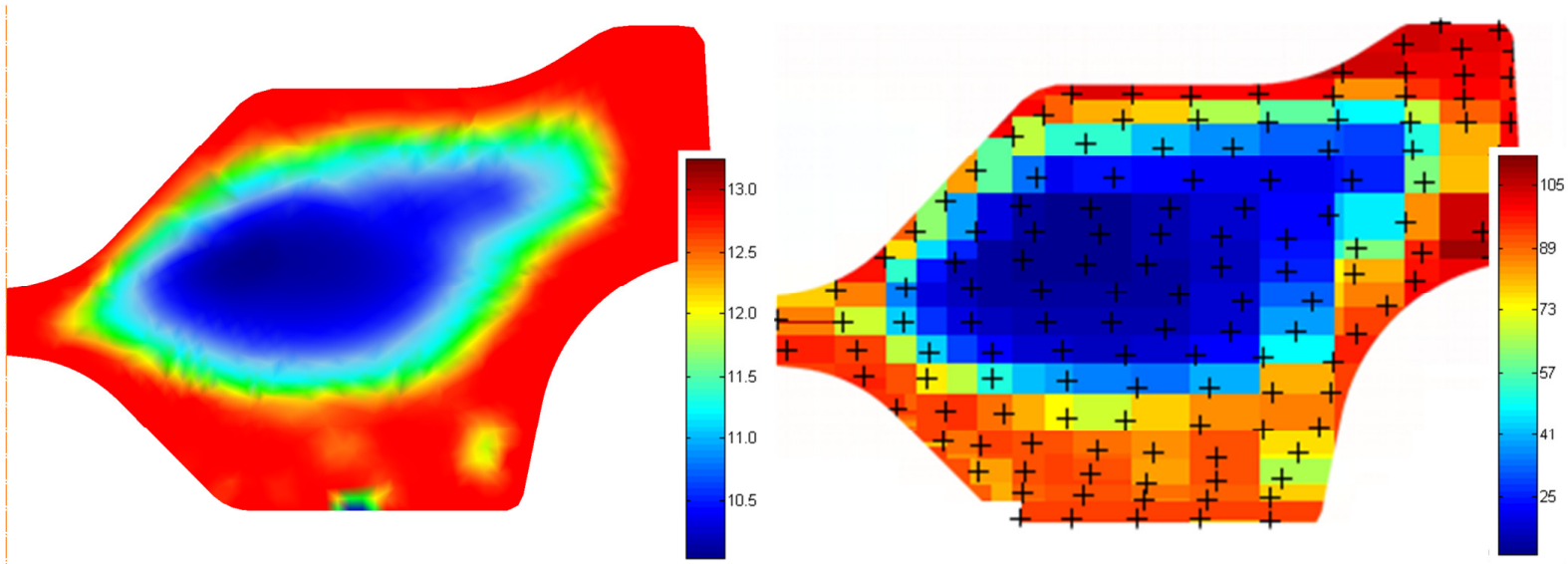

Figure 13. Correlation between grain size $G$ and microstructural energy $e^{*}$ at simulation stage.

The corrected microstructural energy $e^{*}$ maps the simulated grain size with sufficient accuracy. Further on, the obtained factor of heterogeneity $b^{*}$ is shown below for this simulation run.

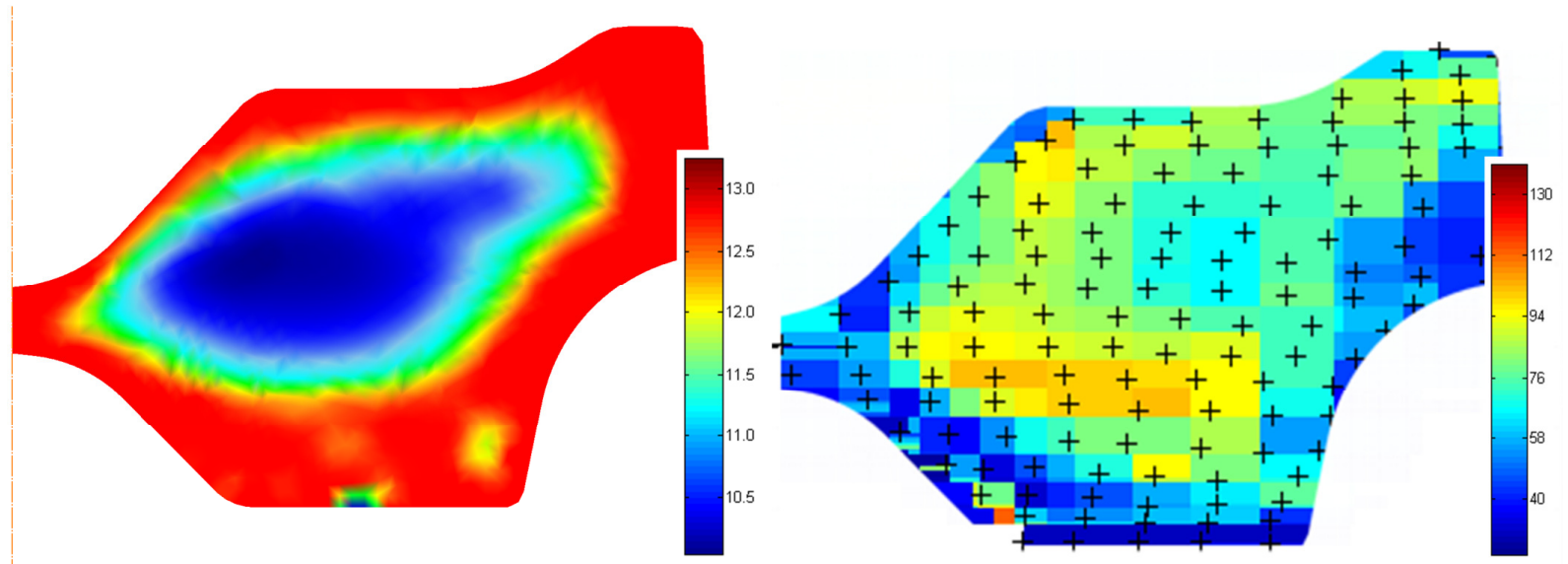

Figure 14. Context of grain size $G$ and factor of heterogeneity $b^{*}$ at simulation stage.

The distribution of the simulated parameters has been verified by comparison with the metallographic sections machined out of cut-ups at defined positions. The mean ASTM grain size $G$ defines the common variable to compare the experimentally ascertained and the simulated microstructural values.

By comparing figure 13 and figure 14 it is clearly visible that the distribution of $e^{*}$ and $b^{*}$ is not similar. As stated earlier, both the microstructural parameters have to be considered for the fatigue life assessment. Figure 15 shows the assessed alternating fatigue life time sustaining at least three million load cycles both graphically and by node-path driven evaluation in radial direction of the forged spool. 

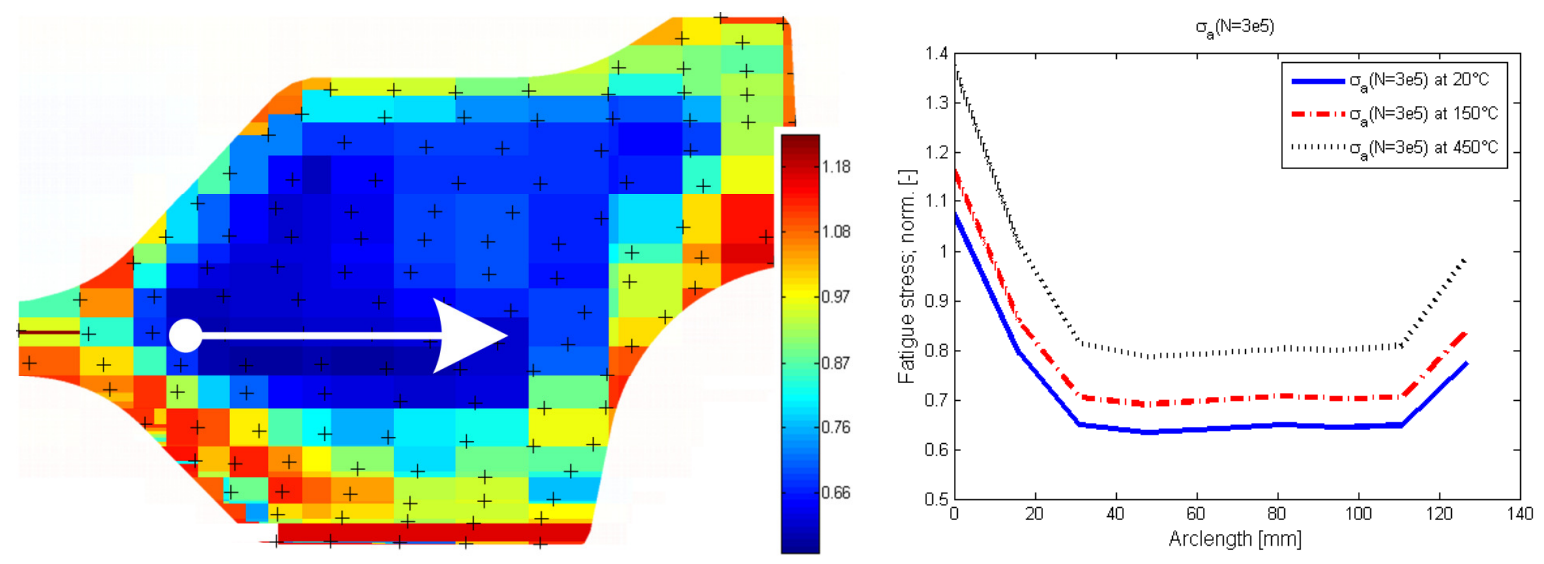

Figure 15. Simulated fatigue stress $\left(150^{\circ} \mathrm{C}\right)$ and path distribution at fatigue region $\left(N_{a}=3 e 5\right)$

The reduction in fatigue stress at the high-cycle endurance region is caused by the decrease in slope of the local SN-curves. The node-path in Figure 16 shows the difference between the finite-life-fatigue and the high-cycle-fatigue region for this specific hot-forging process using the presented closed simulation loop.
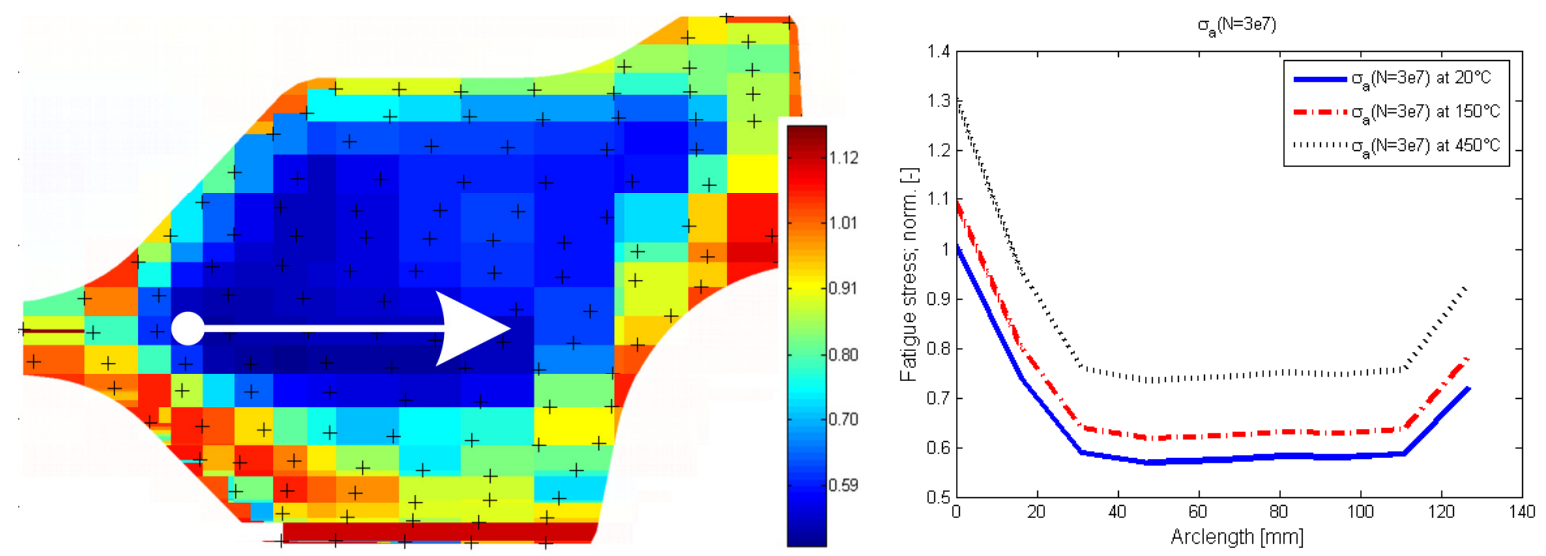

Figure 16. Simulated fatigue stress $\left(150^{\circ} \mathrm{C}\right)$ and path distribution at endurance region $\left(N_{a}=3 e 7\right)$

With the help of the microstructural parameters $e$ and $b$ it is possible to enhance and extend the microstructural model to local fatigue parameters and in the end into the global design chain. 


\section{Conclusion}

The microstructural energy approach introduced here supports an alternative description of the microstructure.

Firstly, the microstructural energy parameter $e$ correlates to the ASTM grain size $G$.

Second, the factor of heterogeneity $b$ characterizes the amount of both non-equiaxed and largesized grains in a unique manner without additional analysis work.

It is therefore possible to basically relate the microstructure to life time behavior. This was achieved by systematic evaluation of numerous fatigue tests carried out at ambient and elevated temperatures and the evaluation of the corresponding metallographic sections. Furthermore, by expansion of the microstructural model introduced by Stockinger with basic numerical findings, corrected values of $e$ and $b$ can be achieved also at simulation stage.

Finally, the entire hot-forging development process including a rough assessment of the local fatigue behavior has been depicted here and can be adapted to aircraft customer superalloy 718 parts on demand.

\section{Acknowledgements}

Financial support by the Austrian Federal Government within the research activities of the Österreichische Forschungsförderungsgesellschaft $\mathrm{mbH}$ acting as Christian Doppler Laboratory for Fatigue Life, operated by the Chair of Mechanical Engineering, Unversity of Leoben, is gratefully acknowledged.

Further on, the Austrian Federal Government and the Styrian Provincial Government, represented by Steirische Wirtschaftsförderungsgesellschaft $\mathrm{mbH}$, within the research activities of the K2 Competence Centre on "Integrated Research in Materials, Processing and Product Engineering", operated by the Materials Center Leoben Forschung GmbH under the frame of the Austrian COMET Competence Centre Programme, is gratefully acknowledged. 


\section{References}

1. B. Pieraggi and J.F. Uginet, "Fatigue and Creep Properties in Relation with Alloy 718 Microstructure", Superalloys 718, 625, 706 and Various Derivatives, (1994), 535-544.

2. E. Andrieu, R. Cozar and A. Pineau, "Effect of Environment and Microstructure on the High Temperature Behaviour of Alloy 718", Superalloy 718-Metallurgy and Applications, (1989), 241-256.

3. J.M. Zhang et.al., "Effect of Hot Deformation Parameters on the Grain Size of Wrought IN718", Superalloys 718, 625, 706 and Various Derivatives, (1997), 183-192.

4. G.E. Korth, "Effects of Various Parameters on the Fatigue Life of Alloy 718", Superalloys 718, 625 and Various Derivatives, (1991), 457-476.

5. L. Shuqi et.al., "The Effect of d-Phase on Crack Propagation under Creep and Fatigue Conditions in Alloy 718", Superalloys 718, 625, 706 and Various Derivatives, (1994), 545-555.

6. P.E. Mosser et.al., "Metallurgical Aspects of Forge Modelling in Alloy 718", Superalloy 718-Metallurgy and Applications, (1989), 179-188.

7. M. Stoschka et.al., "Assessment of Lifetime Calculation of Forged IN718 Aerospace Components Based on a Multi-Parametric Microstructural Evaluation", Superalloys 2008, 573582 .

8. J.F. Uginet and B. Pieraggi, "Study of Secondary Grain Growth on 718 Alloy", Superalloys 718, 625, 706 and Various Derivatives, (1997), 343-352.

9. H. Maderbacher et.al. "Link from the Microstructure to the Fatigue Lifetime of Forged Inconel 718 Components", Danubia-Adria Symposium on Advances in Experimental Mechanics, 26 (2009), 59-60.

10. W. Eichlseder and B. Unger, "Prediction of the fatigue life with the finite element method", SAE Paper, 940245 (1994).

11. N. Kawagaishi, Q. Chen and H. Nisitani, "Fatigue Strength of Inconel 718 at Elevated Temperatures", Fatigue Fracture Engineering Material Structures, 23 (2000), 209-216.

12. M. Stockinger et.al., "Modeling of DELTA-Phase Dissolution During Preheating of INCONEL718 Turbine Disks", Symposium on Superalloys 718, 625, 706 and Derivatives, (2001), 141-148.

13. M. Stockinger and J. Tockner, "Optimizing the Forging of Critical Aircraft Parts by the Use of Finite Element Coupled Microstructure Modeling", Superalloys 718, 625, 706 and Derivatives, (2005), 517-526. 